\title{
A NOTE ON ENTIRE AND MEROMORPHIC FUNCTIONS
}

\author{
S. K. SINGH
}

1. The classical theorem of Borel states that for an entire function $f(z)$ of positive integral order the exponent of convergence of the $a$-points of $f(z)$ is equal to the order of $f(z)$ except possibly for one value of $a$, see Titchmarsh [5, p. 279]. This has been generalized by Nevanlinna [2, p. 77] as follows: there is at most one entire function $g$ such that $\log M(r, g)=o(\log M(r, f))$ and the exponent of convergence of the zeros of $f(z)-g(z)$ is less than the order of $f(z)$. Nevanlinna's proof depends on the second main theorem. We shall give an elementary proof of Nevanlinna's theorem by deducing it from the following result.

TheOREM 1. Let $f(z)=e^{Q_{1}(z)} P_{1}(z)-e^{Q_{2}(z)} P_{2}(z) \not \equiv 0$ where $Q_{1}(z)$ and $Q_{2}(z)$ are polynomials of degree $\rho$ ( $\rho$ being an integer), and $P_{1}(z)$ and $P_{2}(z)$ are canonical products of order $\left\langle\rho\right.$; then $\log M(r, f)>A r^{\rho}$ where $A$ is some suitable positive constant; $M(r, f)$ being $\operatorname{Max}|f(z)|$ for $|z|=r$.

2. Let $n(r)$ denote the number of zeros of $f(z)$ in $|z| \leqq r$, and let

$$
\begin{aligned}
N\left(r, \frac{1}{f-a}\right) & =N(r, a)=\int_{r_{0}}^{r} \frac{n(t, a)}{t} d t, \quad(0 \leqq|a|<\infty) \\
N(r, f) & =N(r, \infty)=\int_{r_{0}}^{r} \frac{n(t, \infty)}{t} d t .
\end{aligned}
$$

We have

THEOREM 2. If $f(z)$ be an entire function of nonintegral order then

$$
\limsup _{r \rightarrow \infty} \frac{N(r, a)}{\log M(r, f)}>0
$$

for all $a,(0 \leqq|a|<\infty)$.

This is a corollary of a theorem of Nevanlinna's [2, p. 51]; cf. Pfluger [3, p. 93]. We shall give a new proof of it. For entire functions of order $\rho<1$ a result better than (2) is known. S. M. Shah [4] and R. P. Boas [1] have proved that for entire functions of order $\rho<1$

Received by the editors October 29, 1956 and, in revised form, April 12, 1957. 


$$
\limsup _{r \rightarrow \infty} \frac{N(r)}{\log M(r, f)} \geqq 1-\rho .
$$

S. M. Shah [4] has also given a similar result for meromorphic functions. It runs:

THEOREM 3. If $F(z)$ be a meromorphic function of order $\rho<1$ then

$$
\limsup _{r \rightarrow \infty} \frac{N(r, a)+N(r, b)}{T(r, F)} \geqq 1-\rho .
$$

For all $a$ and $b, a \neq b,(0 \leqq|a| \leqq \infty ; 0 \leqq|b| \leqq \infty)$.

We shall give a proof of this theorem on the lines of R. P. Boas [1].

3. Proof of Theorem 1. Let

$$
\begin{aligned}
& Q_{1}(z)=a_{1} z^{\rho}+a_{2} z^{\rho-1}+\cdots, a_{1}=\left|a_{1}\right| e^{i \alpha} ; \\
& Q_{2}(z)=b_{1} z^{\rho}+b_{2} z^{\rho-1}+\cdots, b_{1}=\left|b_{1}\right| e^{i \beta}, z=r e^{i \theta},
\end{aligned}
$$

since $P_{1}(z)$ is of order $<\rho$, we can find a set of circles $C_{1}$ of total finite radius such that for $z$ not in $C_{1}, \log \left|P_{1}(z)\right|=o\left(r^{\rho}\right)$. Similarly, if $z$ is not in another such system $C_{2}, \log \left|P_{2}(z)\right|=o\left(r^{\rho}\right)$. If $C=C_{1} \cup C_{2}$, then for $z$ outside $C$,

$$
\left|e^{Q_{1}} P_{1}\right| \geqq e^{\left|a_{1}\right| r^{\rho} \cos (\rho \theta+\alpha)+o\left(r^{\rho}\right)}
$$

and

$$
\left|e^{Q_{2}} P_{2}\right| \geqq e^{\left|b_{1}\right| r^{\rho} \cos (\rho \theta+\beta)+o\left(r^{\rho}\right)}
$$

So

$$
|f| \geqq\left|e^{\left|a_{1}\right| r^{\rho} \cos (\rho \theta+\alpha)+o\left(r^{\rho}\right)}-e^{\left|b_{1}\right| r^{\rho} \cos (\rho \theta+\beta)+o\left(r^{\rho}\right)}\right| .
$$

Now we consider various cases:

If $\left|a_{1}\right| \neq\left|b_{1}\right|$, one of them will be greater than the other and choosing $\theta$ properly, we see that for $z$ outside $C$,

$$
|f| \geqq e^{A_{1} r^{\rho}} \text {. }
$$

If $\left|a_{1}\right|=\left|b_{1}\right|$, but $a_{1} \neq b_{1}$, then $\alpha-\beta \neq 2 k \pi$. So if we choose, $\rho \theta+\alpha$ $=0, \rho \theta+\beta \neq \mathrm{a}$ multiple of $2 \pi$ and hence $\cos (\rho \theta+\beta)<1$. This gives $|f| \geqq e^{A_{1} r^{\rho}}$ in this case also for $z$ outside $C$. Hence $M(r, f) \geqq e^{A_{1} r^{\rho}}$ for a $z$ for which $|z|=r$ does not cut $C$.

Now we can find a finite $d>0$ such that for any $r$, there is a circle with radius $r-t \geqq r-d$, not cutting $C$. Hence

$$
M(r, f) \geqq M(r-t, f) \geqq e^{A_{1}(r-t)^{\rho}} \geqq e^{A_{1}(r-d)^{\rho}} \geqq e^{A r^{\rho}}
$$

finally if $a_{1}=b_{1}$; then 


$$
\begin{aligned}
f(z) & =e^{a_{1} z^{\rho}}\left\{P_{1}(z) e^{a_{2} z^{\rho-1}+\cdots}-P_{2}(z) e^{b_{2} z^{\rho-1}+\cdots}\right\} \\
& =e^{a_{1} z^{\rho}} \psi(z)
\end{aligned}
$$

where $\psi(z)$ is an entire function of order $<\rho$, hence $\log M(r, f) \sim k r^{p}$.

Thus the theorem is proved completely.

Proof of Nevanlinna's Theorem. It is enough, since an entire function of integral order and minimum type cannot have an exceptional value, to prove that if $f(z)$ is of positive integral order $\rho$ there is at most one entire function $g(z)$ such that $\log M(r, g)=o\left(r^{\rho}\right)$ and the exponent of convergence of the zeros of $f(z)-g(z)$ is less than $\rho$. If there are two such functions, $g_{1}$ and $g_{2}$, then

$$
\begin{aligned}
& f-g_{1}=z^{k_{1}} e^{Q_{1}(z)} P_{1}(z), \\
& f-g_{2}=z^{k_{2}} e^{Q_{2}(z)} P_{2}(z)
\end{aligned}
$$

where $Q_{1}(z)$ and $Q_{2}(z)$ are polynomials of degree $\rho$ and $P_{1}(z)$ and $P_{2}(z)$ are canonical products of order $<\rho$. Without any loss of generality we can suppose $k_{1}=k_{2}=0$, then

$$
g_{2}-g_{1}=e^{Q_{1}} P_{1}-e^{Q_{2}} P_{2} .
$$

Hence $\log M\left(r, g_{2}-g_{1}\right)>A r^{\circ}$ by Theorem 1 , and this gives a contradiction.

Proof of Theorem 2. If $r_{n}(a)$ denotes the modulus of the $n$th zero of $f(z)-a$, we know that

$$
\sum\left(\frac{1}{r_{n}(a)}\right)^{\rho+\delta} \text { is convergent for } \delta>0 .
$$

Hence by Valiron's theorem, see Nevanlinna [2, p. 43]

$$
I=\int_{r_{0}}^{\infty} \frac{\log M(r, f)}{r^{\rho+1+\delta}} d r \text { is convergent, }
$$

say $I \leqq M$. Now if possible, let

$$
\lim _{r \rightarrow \infty} \frac{N(r, a)}{\log M(r, f)}=0
$$

then $N(r, a)<(\epsilon / M) \log M(r, f)$ for $r \geqq r_{0}$ so

$$
\begin{aligned}
\int_{r_{0}}^{r} \frac{N(r, a)}{r^{\rho+1+\delta}} d r & <\frac{\epsilon}{M} \int_{r_{0}}^{r} \frac{\log M(r, f)}{r^{\rho+1+\delta}} d r \\
& <\frac{\epsilon}{M} \int_{r_{0}}^{\infty} \frac{\log M(r, f)}{r^{\rho+1+\delta}} d r \\
& <\epsilon .
\end{aligned}
$$


Since the function is of nonintegral order, so $n(r, a) \rightarrow \infty$ as $r \rightarrow \infty$ and hence $N(r, a) \rightarrow \infty$ also as $r \rightarrow \infty$. Thus we can choose $r_{0}^{\prime}\left(\geqq r_{0}\right)$ such that

$$
N\left(r_{0}^{\prime}, a\right)>0
$$

Now

$$
\begin{aligned}
& \epsilon>\int_{r_{0}}^{r} \frac{N(r, a)}{r^{\rho+1+\delta}} d r \geqq \int_{r_{0}}^{r} \frac{N(r, a)}{r^{\rho+1+\delta}} d r \\
& \geqq N\left(r_{0}^{\prime}, a\right) \frac{1}{\rho+\delta}\left[\frac{1}{r_{0}^{\prime} \rho+\delta}-\frac{1}{r^{\rho+\delta}}\right] .
\end{aligned}
$$

First choosing $r$ large enough, and then taking $\epsilon$ arbitrarily small we can make $N\left(r_{0}^{\prime}, a\right)$ arbitrarily small. This contradicts (2). This proves the theorem.

Proof of Theorem 3. Without any loss of generality we can take $a=0, b=\infty$. Let

$$
F(z)=z^{\alpha} \frac{\Pi_{1}(z)}{\Pi_{2}(z)} .
$$

We can further assume $\alpha=0$, then $F(z)$ takes the form

$$
F(z)=\frac{\Pi_{1}(z)}{\Pi_{2}(z)} .
$$

Let $E\left(z / z_{n}, 0\right)=\left(1-z / z_{n}\right)$ be the Weierstrass primary factor corresponding to the zeros of $\Pi_{1}(z)$. Then

$$
\log \left|E\left(\frac{z}{z_{n}}, 0\right)\right|=\log \left|1-\frac{z}{z_{n}}\right| \leqq \log \left(1+\frac{r}{r_{n}}\right) .
$$

So

$$
\begin{aligned}
\log M\left(r, \Pi_{1}\right) & \leqq \sum \log \left(1+\frac{r}{r_{n}}\right)=\int_{0}^{\infty} \log \left(1+\frac{r}{t}\right) d n(t) \\
& \sim r \int_{0}^{\infty} \frac{n(t)}{t(t+r)} d t \\
& \leqq \int_{0}^{r} \frac{n(t, 0)}{t} d t+r \int_{r}^{\infty} \frac{n(t, 0)}{t^{2}} d t .
\end{aligned}
$$

Similarly 


$$
\log M\left(r, \Pi_{2}\right) \leqq \int_{0}^{r} \frac{n(t, \infty)}{t} d t+r \int_{r}^{\infty} \frac{n(t, \infty)}{t^{2}} d t
$$

Now

$$
\begin{aligned}
T(r, F) & \leqq T\left(r, \Pi_{1}\right)+T\left(r, \Pi_{2}\right)+O(1) \\
& \leqq \log M\left(r, \Pi_{1}\right)+\log M\left(r, \Pi_{2}\right)+O(1) \\
& \leqq \int_{0}^{r} \frac{n(t, 0)+n(t, \infty)}{t} d t+r \int_{r}^{\infty} \frac{n(t, 0)+n(t, \infty)}{t^{2}} d t .
\end{aligned}
$$

Set $n_{1}(r)=n(r, 0)+n(r, \infty)$. Then

$$
T(r, F) \leqq \int_{0}^{r} \frac{n_{1}(t)}{t} d t+r \int_{r}^{\infty} \frac{n_{1}(t)}{t^{2}} d t=N_{1}(r)+Q_{1}(r)
$$

say. Now proceeding on the lines of R. P. Boas we get the result.

The author is deeply grateful to Dr. V. Ganapathy Iyer for his help in the preparation of this paper.

\section{REFERENCES}

1. R. P. Boas, Some elementary theorems on entire functions, Rend. Circ. Mat. Palermo vol. I (1952) pp. 1-9.

2. R. Nevanlinna, Fonctions meromorphes, Paris, 1929.

3. A. Pfluger, Zur defektrelation ganzer Functionen end licher Ordnung, Comment. Math. Helv. vol. 19 (1946-1947) pp. 91-104.

4. S. M. Shah, $A$ note on meromorphic functions, Mathematics Student vol. 12 (1944) pp. 67-70.

5. E. C. Titchmarsh, The theory of functions, Oxford, 1952.

D.S. College, Aligarh, India 